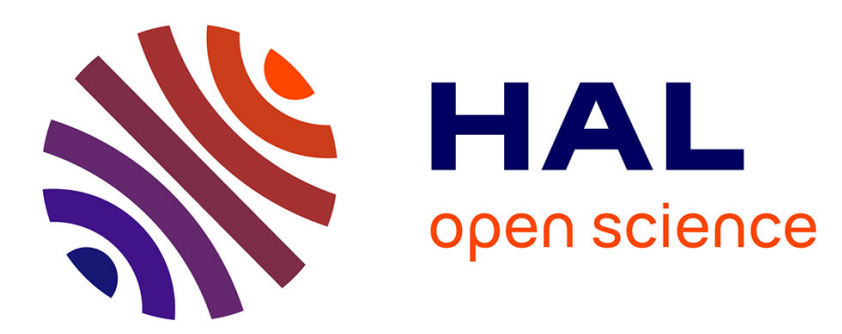

\title{
Elasto-electric coupling for direct electric field distribution measurement in semiconductor structures
}

Basil Salamé, Stéphane Holé

\section{To cite this version:}

Basil Salamé, Stéphane Holé. Elasto-electric coupling for direct electric field distribution measurement in semiconductor structures. Journal of Applied Physics, 2016, 120 (17), pp.175702. 10.1063/1.4966660 . hal-01397095

\section{HAL Id: hal-01397095 \\ https://hal.sorbonne-universite.fr/hal-01397095}

Submitted on 15 Nov 2016

HAL is a multi-disciplinary open access archive for the deposit and dissemination of scientific research documents, whether they are published or not. The documents may come from teaching and research institutions in France or abroad, or from public or private research centers.
L'archive ouverte pluridisciplinaire HAL, est destinée au dépôt et à la diffusion de documents scientifiques de niveau recherche, publiés ou non, émanant des établissements d'enseignement et de recherche français ou étrangers, des laboratoires publics ou privés. 


\title{
Elasto-electric coupling for direct electric field distribution measurement in semiconductor structures
}

\author{
Basil Salamé and Stéphane Holé* \\ Laboratoire de Physique et d'Étude des Matériaux (UMR8213), \\ ESPCI-Paris, PSL Research University, \\ UPMC, Sorbonne Universités, CNRS \\ 10, rue Vauquelin - 75005 Paris - France
}

\begin{abstract}
Semiconductor materials are widely used in electronic industry but their electrical characterization remains complex to estimate without a good model. It has already been shown that an elasto-electric coupling can be used to directly and non-destructively probe the electrical properties at the external interfaces of semi-conductor structures. In this paper it is shown that such a coupling can also be used to probe the inner interfaces of semi-conductor structures. This capability is demonstrated by using a specific semi-conductor structure including a buried silicon p-n junction $720 \mu \mathrm{m}$ away from the external electrodes. The signal generated by the elasto-electric coupling clearly shows separately the electric field at the electrodes and at the buried junction. The contact potential at the buried junction estimated from the measurements is in accordance with the semiconductor doping. This makes it possible to use an elasto-electric coupling for the complete characterization of semiconductor structures.
\end{abstract}

\footnotetext{
* Corresponding author: stephane.hole@espci.fr
} 


\section{INTRODUCTION}

Semiconductor materials are the basic elements of one of the largest industries in the world: the electronic industry. Since the early days, many studies have been carried out to understand the electrical properties of such materials in order to improve on performances [1]. Characterizing these electrical properties requires measurement methods, for instance the measurement of the capacitance or of the current as a function of the applied voltage. These measurement methods are referred to as $\mathrm{C}-\mathrm{V}$ and $\mathrm{I}-\mathrm{V}$ methods respectively $[1,2]$. They are easy to use, relatively cheap and their experimental results can be rapidly obtained. Knowing the signal, it is possible to reconstruct the trapped charge distribution through the material which is a very important information since it controls the device behavior. Indeed many electrical properties of semiconductor materials directly depend on the trapped charge density such as the conductivity [1, 2]. However, the characterization of semiconductor materials with $\mathrm{C}-\mathrm{V}$ and $\mathrm{I}-\mathrm{V}$ methods requires an electrical model of those materials. It is indeed necessary to know the law linking the charge distribution with the capacitance, current and voltage. Therefore these measurement methods are only efficient for well known materials, for control purpose for instance, but are not well adapted for studying new materials since it would require a lot of samples for testing assumptions which is time consuming and very expensive. Moreover the voltage sweep applied during measurements prevents the characterization of semiconductor materials and structures in their real operating conditions.

Direct methods exist for the measurement of space charge distributions. They are mainly used for the characterization of insulating materials under high voltage stress or electron beam irradiation [3,4]. They consist of directly perturbing the charges in the material, either by a local thermal or mechanical perturbation or by a pulsed electric stress. The induced signal then gives an image of the charge distribution $[5,6]$.

Some of these measurement methods have already been used for the characterization of semiconductor materials [7-9]. Though the spatial resolution is not yet sufficient to distinguish the fine structure of the charge profile, it has been shown that they already give precise information [8] and allow measurements under real operating conditions [10]. Contrarily to insulating materials, semiconductor materials contain a lot of mobile carriers. When the material perturbation used to probe the sample acts on the trapped charge density 
during the measurement, the mobile carriers of the semiconductor rearrange themselves as to cancel the electric field far from the material perturbation. The distance on which the electric field is canceled is called the screening length $\lambda$ and is of the order of tens of nanometers in semiconductor materials. Close to the interfaces, that is to say at a distance smaller than $\lambda$, the screening is not complete and a signal is produced $[8,10]$. However far from the interfaces, it was not clear whether a signal could be obtained.

In this paper it is shown that a signal can be obtained from a semiconductor bulk, in particular at a buried junction, by using an elasto-electric coupling. In the first section the Pressure-Wave-Propagation (PWP) method, which is an elasto-electric coupling method for measuring space charge distributions, is briefly introduced. In the second section the sample is described and validated as to prevent signal misinterpretations. In the third section, results are exposed and discussed before conclusion.

\section{PWP METHOD PRINCIPLE}

The Pressure-Wave-Propagation (PWP) method [6, 11] is an elasto-electric coupling method for the direct and non-destructive electrical characterization of insulators. An elastic wave is transmitted to the material to be tested. During its propagation at the velocity of sound, the elastic wave moves the trapped charges encountered which generates in turn an electric signal between adjacent electrodes. When the elastic wave is a unipolar pressure pulse, it locally moves the charges encountered at a speed $\partial u / \partial t$, where $u$ is the material displacement produced by the pressure pulse. Under short-circuit conditions, the local charge displacement gives birth to a current $\rho A \partial u / \partial t$, where $\rho$ is the charge density at the position of the pressure pulse and $A$ is the sample tested area. As a result, the short-circuit current at a time $t$ is proportional to the charge density at the position $x$ of the pressure pulse inside the sample, time and position being simply connected by the velocity of sound $v_{s}$. Under open-circuit conditions however, the current is prevented to flow. Hence, each charge displacement since the entrance of the pressure pulse inside the sample produces an electric field variation $\delta E=\rho u / \epsilon$, where $\epsilon$ is the permittivity. As the associated voltage variation appearing across sample is proportional to the integral of $\delta E$ over space, it is also proportional to the integral of the charge density over space and therefore to the electric field at the position of the pressure pulse. That is illustrated in Figure 1. 
As a measured signal can be the image of the charge density or of the electric field, it is important to check the measurment conditions prior to any analysis. For that purpose, a resistor $R$ is added in series with the sample before the amplifier (see the difference between figures $2 \mathrm{a}$ and $2 \mathrm{~d}$ ). As a consequence the cutoff frequency of the measurement system is shifted toward a lower frequency (see the difference between figures $2 \mathrm{~b}$ and $2 \mathrm{e}$, and between figures $2 \mathrm{c}$ and $2 \mathrm{f}$ ). If measurements are carried out in open-circuit conditions, the signal is multiplied by the factor of the bridge made by the resistor $R$ and the 50 - $\Omega$-input impedance of the amplifier. If measurements are carried out in short-circuit conditions, the signal has the same amplitude because $R$ is in the current loop. Eventually the signal can slightly change in shape due to the modification of the cutoff frequency.

In this study the pressure pulse is generated by the impact of a high-power nanosecondduration laser pulse (100 MW during $5 \mathrm{~ns}$ ) that strikes an aluminum target acoustically coupled with phenyl salicylate to one side of the vacuum deposited electrodes of the sample. Sample electrodes are connected to an oscilloscope via a 50- $\Omega$-input-impedance $400-\mathrm{MHz}$ bandwidth 40-dB amplifier.

\section{SAMPLE PREPARATION AND VALIDATION}

Samples consist of two differently doped semiconductor wafers bonded together and vacuum deposited electrodes on both external faces. This sample structure presents thus 3 junctions, a Schottky junction at both external interfaces and a p-n junction in the bulk of the sample. It is thus a convenient structure to assess the detection capability of the elasto-electric coupling measurement technique.

\section{A. Sample preparation}

Two $720-\mu$ m-thick silicon wafers, one n-doped (4 to $7 \Omega / \mathrm{cm})$ and the other p-doped (1 to $50 \Omega / \mathrm{cm}$ ) are bonded together by molecular bonding [12]. As a consequence a buried junction is created $720 \mu \mathrm{m}$ away from the external interfaces. A thermal treatment at $900^{\circ} \mathrm{C}$ is used to produce Si-Si covalent bonds. After the thermal treatment, the bonded wafers are cut into $25 \mathrm{~mm} \times 25 \mathrm{~mm}$ pieces and 20 -mm-diameter aluminum or platinum electrodes are vacuum deposited by sputtering on each side. 


\section{B. Sample validation}

It is necessary to ensure that an elastic continuity exists at the buried junction between the two bonded wafers. Indeed any elastic discontinuity would result in wave reflection and thus could prevent a clear and definitive interpretation of the obtained signals. In order to verify the elastic continuity of our samples, we have used two 50-MHz ultrasonic transducers (Sofranel VF502FS) acoustically coupled with aqueous gel (Sofranel gel D) on each side of a sample. Ultrasonic pulses are emitted from one side and received at the other side. Since elastic discontinuities produce echoes, the delays between the ultrasonic pulses arriving at the receiving transducers give information on the location of elastic discontinuities. In the case of an elastic continuity at the buried junction, reflections occur only at external interfaces. The delay between echoes is then equal to twice the transit time through the sample (Figure 3a). In the case of an elastic discontinuity at the buried junction, reflections occur both at external interfaces and at the buried junction. The delay between echoes is then equal to the transit time through the sample (Figure $3 \mathrm{~b}$ ).

The transit time through the sample can be estimated by successively measuring the delay of the direct ultrasonic pulse, that is to say the first pulse in the signal, when there is no sample between the transducers (Figure 4a) and when there is a sample between the transducers (Figure 4b). Measurements are averaged when there is a sample between the transducers in order to reduce the noise level and then to detect lower amplitude signals. Results presented in Figure 4 are similar for all samples. It can be deduced that transit time $\Delta t$ is $171.5 \mathrm{~ns}$ and that delay between echoes is twice that transit time. As a consequence it can be concluded that no significant reflection occurs at the buried junction and thus that samples are acoustically continuous. Knowing the sample thickness, the velocity of sound is estimated to $v_{s}=8397 \mathrm{~m} / \mathrm{s}$, which is consistent with the theoretical values, between $8388.3 \mathrm{~m} / \mathrm{s}$ in doped silicon and $8432.8 \mathrm{~m} / \mathrm{s}$ in pure silicon [13].

\section{PWP MEASUREMENTS AND DISCUSSION}

Figure 5a shows a PWP signal obtained with one of the samples. It exhibits 3 peaks. The first peak is generated when the pressure pulse enters the sample. The second peak, which is about $85 \mathrm{~ns}$ after the first one, is generated by the pressure pulse at the buried 
Table I. Peak amplitude in Figure 5a, Figure 6a and Figure 6b.

\begin{tabular}{|c|c|c|c|}
\hline Amplitude $(\mathrm{mV})$ for & First peak & Second peak & Third peak \\
\hline \hline Figure 5a & $32.7 \pm 0.7$ & $3.9 \pm 0.7$ & $29.4 \pm 0.7$ \\
\hline Figure 6a & $5.2 \pm 0.7$ & $4.9 \pm 0.7$ & $6.2 \pm 0.7$ \\
\hline Figure 6b & $6.2 \pm 0.7$ & $5.0 \pm 0.7$ & $3.3 \pm 0.7$ \\
\hline
\end{tabular}

junction. The third peak, which is about $170 \mathrm{~ns}$ after the first one, is generated when the pulse leaves the sample.

The measurement conditions are determined by inserting a resistor $R=150 \Omega$ in series with the sample before the amplifier as illustrated in Figure 2d. The result presented in Figure 5b clearly shows that measurements are carried out in open-circuit conditions. Indeed the signal is reduced by a factor 4 which corresponds to the bridge factor coefficient 50/(50+ $R)=0.25$. As a consequence, signal has to be interpreted as an electric field distribution, each nanosecond corresponding to $1 \mathrm{~ns} \times v_{s}=7.86 \mu \mathrm{m}$ in the material.

The first and third peaks in the signal are due to the Schottky diodes made by the metalsemiconductor interface at both sides of the sample [8]. The second peak, which is clearly visible, is due to the buried junction. Amplitude of the peaks are reported in Table I. In order to ensure that the second peak in the signal is not due to an artifact and does not depend on electrode material, aluminum $(\mathrm{Al})$ is replaced by platinum $(\mathrm{Pt})$ and measurements are carried out in both directions of the sample, that is to say when the pressure pulse goes from $\mathrm{n}$ to $\mathrm{p}$ and from $\mathrm{p}$ to $\mathrm{n}$.

It can be seen in Figure 6a that using platinum electrodes instead of aluminum electrodes alters the interface peak amplitude but not the buried junction peak amplitude (see Table I). The work function of $\mathrm{Pt}$ is indeed larger than the one of $\mathrm{Al}$, which changes the Schottky barrier at the electrodes interfaces [8]. In addition, when the sample is flipped so that the measurement is carried out with a pressure pulse propagating in the other direction, Figure $6 \mathrm{~b}$, interfacial peaks do not change but the one at the buried junction does. The direction of the electric field at the junction has indeed been flipped whereas the symmetry of the sample structure does not effect the interface structure, i.e. metal first at one side and metal last at the other side.

The amplitude of the peaks at the interface is proportional to the contact potential be- 
tween the metal and the semiconductor because the pressure pulse extent is much larger than the electric field extent. This contact potential depends on the electrode work function, semiconductor affinity, semiconductor doping, but also on surface state density (see section 5.5.1 in [1]). Taking into account the peak amplitudes and the work function of the electrodes, $4.28 \mathrm{eV}$ for $\mathrm{Al}$ and $5.65 \mathrm{eV}$ for $\mathrm{Pt}$ [14], and also a surface state coefficient of the order of 0.36 [8], measurements can be calibrated as $(5.65-4.28) /(0.36 \times(32.7-5.2))=$ $0.138 \mathrm{eV} / \mathrm{mV}$. The contact potential at the buried junction can therefore be estimated to $0.138 \times(3.9+4.9) / 2=0.61 \mathrm{~V}$. This value can be compared to the one calculated from each wafer doping using the relation between resistivity and impurity concentration (see sections 1.5.2 and 2.3.1 in [1]). One obtains a concentration from 0.57 to $1 \times 10^{15} \mathrm{~cm}^{-3}$ for the ndoped wafer and from 0.2 to $10 \times 10^{15} \mathrm{~cm}^{-3}$ for the $\mathrm{p}$-doped silicon wafer, which leads to a contact potential between 0.55 and $0.66 \mathrm{~V}$ at room temperature. This is consistant with the measured results considering doping uncertainties. Notice that an estimation of the contact potential with the $\mathrm{C}-\mathrm{V}$ method would have been biased by the interface Schottky diodes in series with the buried junction.

Thus, it appears that, in contrast with what one may think, the signal is not completely screened far from the interfaces. In fact when the pressure pulse enters the buried junction, trapped charges in the space charge region are compressed which results in a variation of the electric field distribution. However, there is still the same amount of trapped charges on both sides of the junction and therefore, the electric field remains zero outside the space charge region. As a consequence mobile carriers do not move, so no screening occurs. And as the integral of the electric field distribution has changed, a voltage variation is detected across the sample. This is illustrated in Figure 7. Finally the signal obtained at the buried junction is an image of the electric field at that junction. Of course the duration of the pressure pulse is not yet sufficiently short to resolve the fine structure of the electric field distribution but is already sufficiently short to separate interface and buried junction signals of the semiconductor structure. It is then possible to characterize independently and at the same time these regions of the semiconductor structure contrarily to C-V and I-V methods that give a single signal over the whole semiconductor structure without any discernment. 


\section{CONCLUSION}

In this paper we have shown that the Pressure-Wave-Propagation (PWP) method can also be applied for studying semiconductor structures containing buried interfaces. Experimental measurements have been carried out with p-n junctions made of two wafers molecularly bonded together. This sample structure has been specifically designed to assess the capability of the measurement technique. The elastic continuity of the structure has been tested to avoid misinterpretation of the PWP signals. Though at $720 \mu \mathrm{m}$ away from electrodes, there is no effect of the screening on the buried junction signal which can be directly interpreted as the electric field distribution through the structure in open-circuit measurement conditions. The estimated voltage barrier from the signal is consistent with the doping used. Contrarily to indirect measurement techniques, the elasto-electric coupling measurement method can discriminate the influence of the various regions of a semiconductor structure. As no electrical model is required, the elasto-electric coupling measurement method already offers a powerful tool to study new semiconductor materials ans structures.

\section{ACKNOWLEGMENT}

The authors thank Frank Fournel from CEA-LETI (Grenoble, France) for providing us with the wafer molecular bonding and sample cutting.

[1] S.M. Sze. Semiconductor devices: physics and technology. John Wiley \& Sons, USA, 2001. ISBN: 0471333727.

[2] E.H. Nicollian and J.R. Brews. MOS (Metal Oxide Semiconductor) physics and technology. John Wiley \& Sons, USA, 1982. ISBN: 0471085006.

[3] M. Fu, G. Chen, L.A. Dissado, and J.C. Fothergill. Influence of thermal treatment and residues on space charge accumulation in XLPE for DC power cable application. IEEE Trans. Dielectr. E.I., 14:53-64, 2007.

[4] S. Le Roy, F. Baudoin, V. Griseri, C. Laurent, and G. Teyssedre. Charge transport modelling in electron-beam irradiated dielectrics: a model for polyethylene. J. Phys. D:Appl. Phys., 43:315402, 2010 . 
[5] T. Mizutani. Space-charge measurement techniques and space-charge in polyethylene. IEEE Trans. Dielectr. EI., 1:923-933, 1994.

[6] S. Holé, T. Ditchi, and J. Lewiner. Non-destructive methods for space charge distribution measurements: what are the differences? IEEE Trans. Dielectr. E.I., 10:670-677, 2003.

[7] S. Holé, T. Ditchi, and J. Lewiner. Can non-destructive space charge measurement techniques have fallout in other fields? In 11th International Symposium on Electrets, pages 32-35, Melbourne, Australia, 2002. IEEE. DOI: 10.1109/ISE.2002.1042938.

[8] C. Ravat, É. Absil, S. Holé, and J. Lewiner. Acoustoelectric coupling for direct electrical characterization of semiconductor devices. J. Appl. Phys., 99:063712-1-5, 2006.

[9] L. Boyer, O. Fruchier, P. Notingher, S. Agnel, A. Toureille, B. Rousset, and J.L. Sanchez. Analysis of data obtained using the thermal-step method on a MOS structure - an electrostatic approach. IEEE Trans. Ind. Appl., 46:1144-1150, 2010.

[10] S. Holé and J. Lewiner. Characterization of semiconducting structures by the PWP method. In 13th International Symposium on Electrets, page B0302, Tokyo, Japan, 2008. IEEE. DOI: 10.1109/ISE.2008.4813999.

[11] C. Alquié, G. Dreyfus, and J. Lewiner. Stress wave probing of electric field distributions in dielectrics. Phys. Rev. Lett., 47:1483-1487, 1981.

[12] F. Fournel, H. Moriceau, B. Aspar, K. Rousseau, J. Eymery, J.L. Rouviere, and N. Magnea. Accurate control of the misorientation angles in direct wafer bonding. Appl. Phys. Lett., 80:793-795, 2002.

[13] J.J. Hall. Electronic effects in the elastic constants of n-type silicon. Phys. Rev., 161:756-761, 1967. DOI: http://dx.doi.org/10.1103/PhysRev.161.756.

[14] H. B. Michaelson. The work function of the elements and its periodicity. J. Appl. Phys., 48:4729-4733, 1977. 
Figure 1. PWP method principle. A laser pulse produces a pressure pulse that moves the charges encountered and induces an electrical signal correlated with the electric field distribution inside the sample.

Figure 2. Identification of the measurement conditions. (a) Measurement set-up, equivalent circuit and corresponding frequency response in (b) short-circuit and (c) open-circuit conditions. (d) Measurement set-up with a resistor in series, equivalent circuit and corresponding frequency response in (e) short-circuit and (f) open-circuit conditions. In open-circuit conditions, the amplitude of the signal is reduced by the bridge factor contrarily to short-circuit conditions.

Figure 3. Ultrasonic echoes in a position-time diagram. (a) In the case of elastic continuity, the delay between echoes is twice the transit time $\Delta t$ in the sample. (b) In the case of elastic discontinuity, the delay between echoes is equal to the transit time $\Delta t$ in the sample.

Figure 4. Validation tests. (a) Measurement without sample between transducers. (b) Averaged measurement with a sample between transducers, where $\Delta t$ corresponds to the transit time. Echoes in the sample are separated by twice the transit time.

Figure 5. (a) Measurement of a p-n junction bonded at $900^{\circ} \mathrm{C}$ with aluminum electrodes. (b) Determination of the measuring conditions using a 150- $\Omega$-resistor in series. The signal is divided by 4 , then open-circuit conditions apply.

Figure 6. Measurements of a p-n junction with platinum electrodes. The pressure pulse propagates (a) from $n$ to $\mathrm{p}$ and (b) from $\mathrm{p}$ to $\mathrm{n}$.

Figure 7. Schematic process involved at the origin of the signal generated at the buried junction. (a) Before the pressure enters the space charge region. (b) The pressure pulse compresses the space charge region. The electric field distribution is modified but remains zero outside the space charge region. Mobile carriers do not move (no screening) and a voltage variation $v_{m}$ is generated. 
Basil Salamé - Figure 1

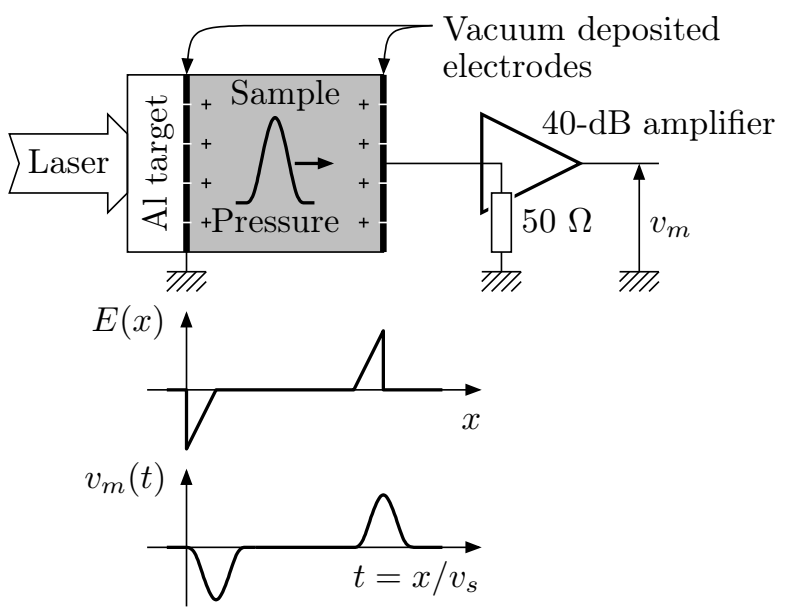


Basil Salamé - Figure 2

(a)

(d)
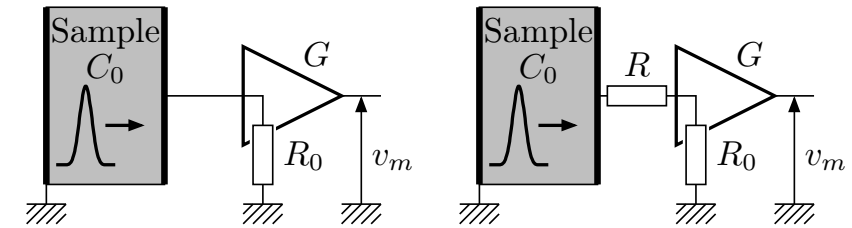

(b)

(c)
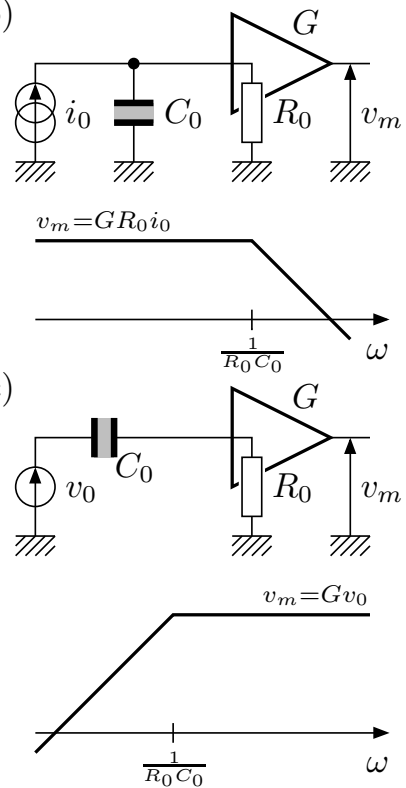

(e)

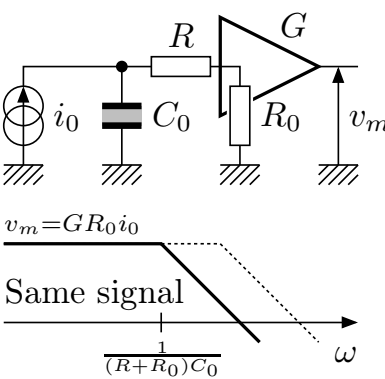

(f)

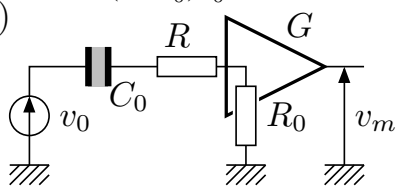


Basil Salamé - Figure 3

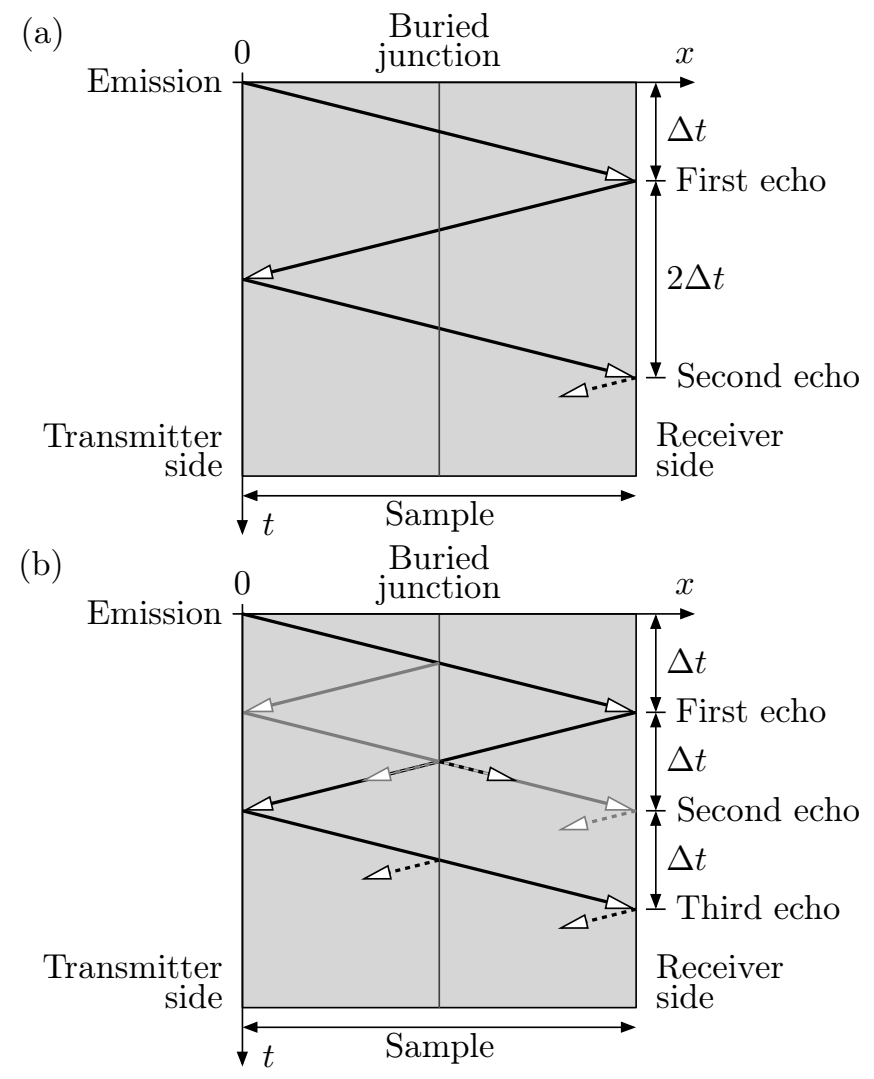


Basil Salamé - Figure 4

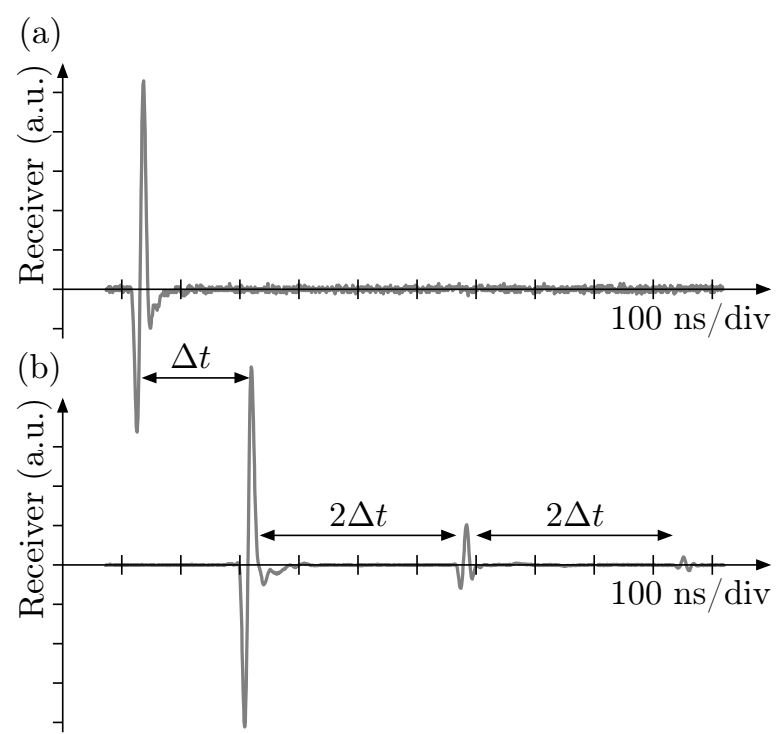


Basil Salamé - Figure 5

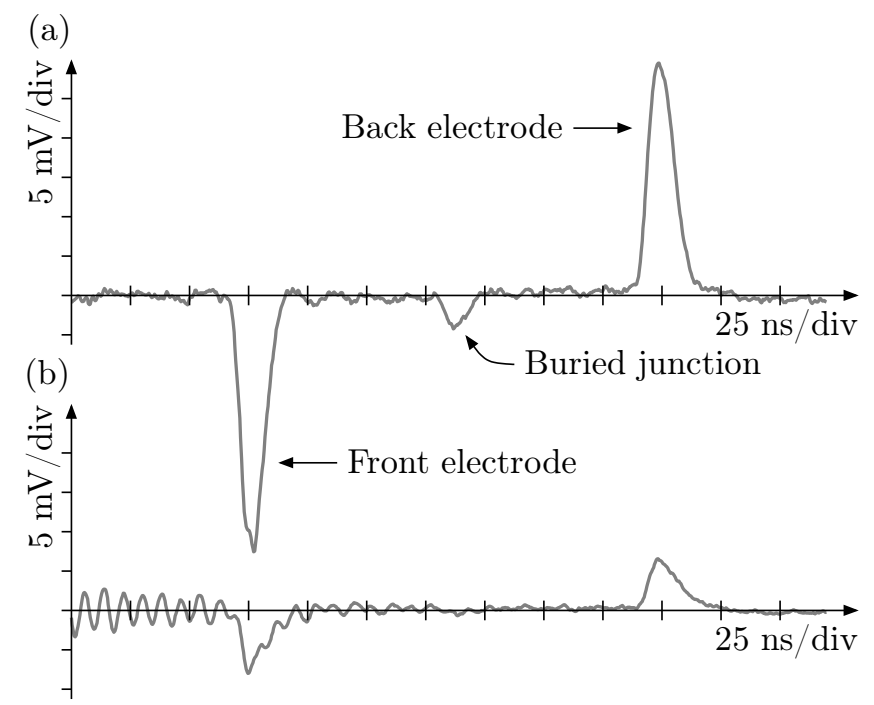


Basil Salamé - Figure 6

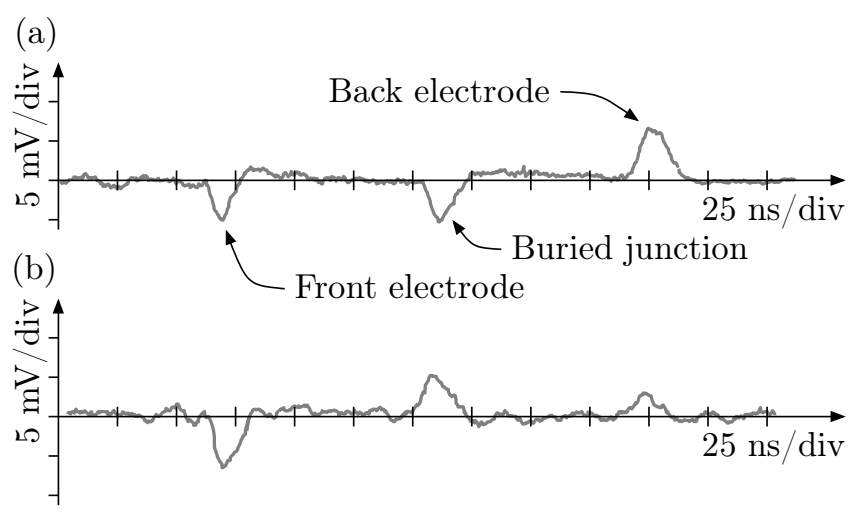


Basil Salamé - Figure 7

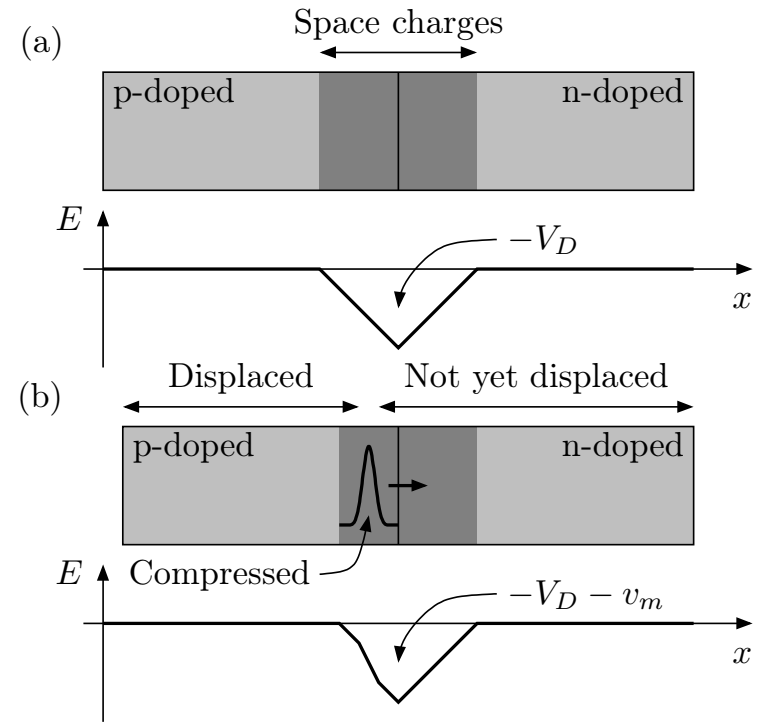

